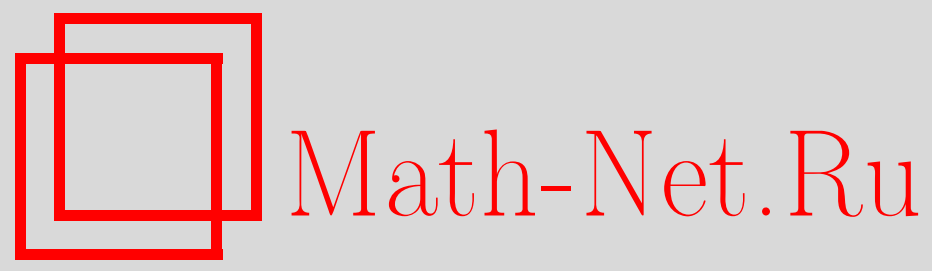

М. Х. Аль-Совейл, Рандомизированный алгоритм множественного выбора, Дискрет. матем., 2006, том 18, выпуск $3,95-101$

DOI: https://doi.org/10.4213/dm62

Использование Общероссийского математического портала Math-Net.Ru подразумевает, что вы прочитали и согласны с пользовательским соглашением http://www . mathnet.ru/rus/agreement

Параметры загрузки:

IP : 3.82 .47 .9

26 апреля 2023 г., $10: 55: 24$

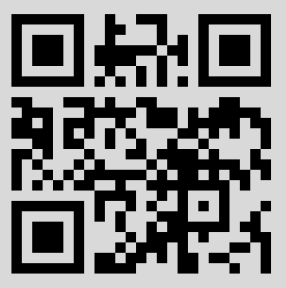




\title{
Рандомизированный алгоритм множественного выбора
}

\author{
() 2006 г. М. Х. Аль-Совейл
}

\begin{abstract}
Пусть заданы множество $S$, состоящее из $n$ элементов линейно упорядоченного множества, и множество $K=\left\{k_{1}, k_{2}, \ldots, k_{r}\right\}$ различных положительных целых чисел, $1 \leqslant k_{1}<k_{2}<\ldots<k_{r}<n$. Задача множественного выбора состоит в нахождении $k_{i}$-х наименьших элементов множества $S$ для всех $i=1, \ldots, r$. В работе предложен эффективный рандомизированный алгоритм, решающий эту задачу за время $O(n \ln r)$ с вероятностью, не меньшей $1-c n^{-1}$, где $c$ - положительная постоянная. Алгоритм можно рассматривать как единообразный подход к задачам рандомизированного выбора, множественного выбора и сортировки.
\end{abstract}

\section{1. Введение}

Пусть $S$ - множество, состоящее из $n$ элементов некоторого линейно упорядоченного множества, и $K=\left\{k_{1}, k_{2}, \ldots, k_{r}\right\}-$ множество целых положительных чисел, $1 \leqslant k_{1}<k_{2}<\ldots<k_{r} \leqslant n$, которое будем называть множеством рангов. Задача множественного выбора состоит в нахождении $k_{i}$-х наименьших элементов множества $S$ для всех $i=1, \ldots, r$. При $r=n$ задача множественного выбора представляет собой задачу сортировки.

Нахождение эффективных алгоритмов для решения задачи множественного выбора получило больше внимания не со стороны сторонников последовательного анализа, а со стороны тех, кто занимается параллельными вычислениями. Классический простой последовательный алгоритм из [6] остается единственным и является базовым для ряда параллельных алгоритмов, разработанных на его основе. По-видимому, первый пример параллелизации задачи множественного выбора содержится в [9], где предложен оптимальный параллельный алгоритм с рабочим временем $O\left(n^{\varepsilon} \log r\right)$ на EREW PRAM с $n^{1-\varepsilon}$ процессорами, $0<\varepsilon<1$. В случае, когда $\varepsilon=\log \left(\log n \log { }^{*} n\right) / \log n$, время работы этого алгоритма есть $O\left(\log n \log ^{*} n \log r\right)$. В [9] предложена общая схема параллелизации оптимального последовательного алгоритма, который вначале находит элемент $x$ ранга $k_{r / 2}$, разбивающий $S$ на две группы, состоящие соответственно из элементов, меньших $x$, и элементов, больших $x$, и тем самым приводит к двум подзадачам, которые решаются рекурсивно применением алгоритма. Другой алгоритм с теми же временем работы и числом процессоров предложен в [2]. Этот алгоритм является простой модификацией параллельного алгоритма сортировки из [1]. В [3] предложен наиболее эффективный параллельный алгоритм. Его время работы на EREW PRAM с $p, r \leqslant p<n$, процессорами есть $O\left(\left((n / p)+t_{s}(p)\right)(\lg r+\lg (n / p))\right)$, где $t_{s}(p)-$ время, требуемое для сортировки $p$ 
элементов $p$ процессорами. Другие результаты, связанные с алгоритмом из [9], можно найти в [10]-[13].

В настоящей статье делается попытка, перейдя на позиции последовательного анализа, представить простой в описании, эффективный и быстрый рандомизированный алгоритм. В его основе лежит ясная интуитивная идея, и алгоритм допускает довольно простой анализ.

\section{2. Детерминированный алгоритм множественного выбора}

Алгоритм множественного выбора MULTISELECT из [6], примененный к задаче с параметрами $(S, K)$, состоит в следующем. Находим $\lceil k / 2\rceil$-й элемент $a$, разбиваем исходное множество $S$ на два множества $S_{1}$ и $S_{2}$, состоящие, соответственно, из элементов, меньших и больших $a$, и переходим к двум задачам, одной с $S_{1}$ и $\left\{k_{1}, k_{2}, \ldots,\lceil k / 2\rceil\right\}$ и другой с $S_{2}$ и $\left\{\lceil k / 2\rceil+1,\lceil k / 2\rceil+2, \ldots, k_{r}\right\}$. Приведем краткое описание алгоритма в задаче с параметрами $(S, K)$.

Алгоритм MultiSELECT. 1. Если $|K|>0$, выполняем шаги 2-6.

2. Полагаем $k=k_{[r / 2]}$. Для нахождения $k_{\lceil r / 2\rceil}$-го элемента $s \in S$ используем алгоритм сортировки (SELECT) элементов $S$.

3. Сравнивая $s$ с элементами множества $S$, строим множества $S_{1}$ и $S_{2}$ элементов, соответственно, меньших и больших $s$.

4. Полагаем $K_{1}=\left\{k_{1}, k_{2}, \ldots, k_{[r / 2\rceil}\right\}, K_{2}=\left\{k_{[r / 2\rceil+1}, k_{[r / 2\rceil+2}, \ldots, k_{r}\right\}$.

5. Применяем алгоритм MULTISELECT к задаче с параметрами $\left(S_{1}, K_{1}\right)$.

6. Применяем алгоритм MULTISELECT к задаче с параметрами $\left(S_{2}, K_{2}\right)$.

На шаге 2 применяем детерминированный алгоритм SELECT с временем работы $\Theta(n)$. Ясно, что алгоритм решает задачу множественной сортировки за время $\Theta(n \log r)$, так как глубина рекурсии есть $\log r$ и работа, проводимая на каждом уровне рекурсивного дерева, требует время $\Theta(n)$. Что касается оценки снизу для алгоритма множественного выбора, предположим, что она есть $o(n \log r)$. Тогда, полагая $r=n$, мы получили бы возможность сортировки $n$ элементов за время $o(n \log n)$, что противоречило бы известной оценке $\Omega(n \log n)$. Эта нижняя оценка была получена в [6]. Итак, сложность задачи множественного выбора есть $\Omega(n \log r)$, так что описанный выше алгоритм является оптимальным.

Детерминированный алгоритм множественного выбора, описанный выше, так же, как любой другой детерминированный алгоритм, есть алгоритм типа известного классического алгоритма выбора, предложенного в [4]. Алгоритм MULTISELECT мало применим на практике, особенно при небольших значениях $n$. Эта непрактичность наследуется от классического последовательного алгоритма множественного выбора. Чтобы убедиться в этом, рассмотрим случай, когда $K=\{1,2,3\}$. Алгоритм вначале привлекает алгоритм SELECT с входом $S$, чтобы найти второй наименьший элемент. При следующем привлечении алгоритма SELECT к $n-2$ оставшимся элементам из $S$ выбирается третий по величине элемент. В общем случае с использованием рекурсивного дерева можно показать, что если 
$K=\{1,2, \ldots, r\}$, то рассматриваемый алгоритм привлекает алгоритм SELECT $(\log n \log r)$ раз к задачам с по меньшей мере $n-r-1$ элементами.

Алгоритм, описанный в [7], который мы будем называть алгоритмом FIND, является популярным детерминированным алгоритмом выбора в силу его простоты и хорошим в среднем временем работы, несмотря на то, что его наихудшее время есть $O\left(n^{2}\right)$. Повидимому этот алгоритм является лучшим кандидатом для использования в сочетании с алгоритмом MULTISELECT.

Очевидной альтернативой для улучшения эффективности алгоритма является использование рандомизации. Алгоритм QUICKSELECT, являющийся рандомизированной версией алгоритма FIND, будет хорошей заменой алгоритма SELECT на втором шаге. Однако случаи, когда ранги сосредоточены в одной или более областях, особенно в двух крайних областях, как в рассмотренном выше примере, представляют собой особые случаи. Если ранги сосредоточены в начале, например, если $K=\{1,2, \ldots, r\}$, то было бы желательно избавляться от как можно большего числа нежелательных больших элементов.

\section{3. Алгоритм}

В этой статье мы предлагаем простой алгоритм, который хорошо приспособлен для решения задачи множественного выбора. Рандомизированный QUICKSORT является мощным алгоритмом, и как показано в [8], многие геометрические алгоритмы объединяются использованием в той или иной степени схемы разбиения алгоритма QUICKSORT. Задача множественного выбора также не является исключением, небольшая модификация алгоритма QUICKSORT эффективно решает задачу множественного выбора. Идея модификации проста. Назовем элементы, которые ищутся в задаче множественного выбора, целями. Например, если $j \in K$, то $j$-й наименьший элемент в $S$ есть цель. Выберем элемент $s$ случайно равновероятно из $S$ и разобьем элементы $S$ на малые и большие элементы относительно $s$. Если множества как малых, так и больших элементов содержат цели, то пусть QUICKSORT продолжает работать обычным образом. В противном случае, если только малые (большие) элементы содержат цели, то отбрасываем большие (малые) и обращаемся к малым (большим) элементам. Таким образом, алгоритм есть гибрид алгоритмов QUICKSORT и QUICKSELECT. Заметим, что здесь под алгоритмом QUICKSORT понимается рандомизированная версия этого алгоритма.

При описании предлагаемого алгоритма будут использоваться следующие обозначения для повторных разбиений множества $S$ на более мелкие множества. Пусть $y \in S$ и ранг $k_{y} \in K$. Разобьем $S$ на подмножества

$$
S_{\leqslant}=\{x \in S \mid x \leqslant y\}, \quad S_{>}=\{x \in S \mid x>y\} .
$$

Это разбиение множества $S$ индуцирует разбиение множества $K$ на подмножества

$$
K_{\leqslant}=\left\{k \in K \mid k \leqslant k_{y}\right\}, \quad K_{>}=\left\{k-k_{y} \mid k \in K, k>k_{y}\right\} .
$$

Пары $\left(S_{\leqslant}, K_{\leqslant}\right)$и $\left(S_{>}, K_{>}\right)$будем называть селекционными парами. Селекционная пара $(S, K)$, а также множества $S$ и $K$ называются активными, если $|K|>0$, в противном случае они называются пассивными. Предлагаемый рандомизированный алгоритм множественного выбора назовем алгоритмом QUICK-MULTISELECT, он может быть описан следующим образом. 
Алгоритм QUICK-MULtiSELECT (для пары $(S, K)$ ). 1. Если $|K|>0$, то выполняем шаги $2-6$.

2. Если $\{a\}$ и $|K|=1$, то выход равен $a$.

3. Пусть $s$ - элемент, выбранный случайно с равными вероятностями из $S$.

4. Сравнивая $s$ с элементами $S$, определяем множества $S_{\leqslant}$и $S_{>}$элементов, не превосходящих $s$ и больших $s$, соответственно. Вычисляем ранг $r(s)$ элемента $s \in S$. Используя $r(s)$, разбиваем $K$ на $K_{\leqslant}$и $K_{>}$.

5. Если $\left|K_{\leqslant}\right|>0$, то применяем QUICK-MULTISELECT к паре $\left(S_{\leqslant}, K_{\leqslant}\right)$.

6. Если $\left|K_{>}\right|>0$, то применяем QUICK-MULTISELECT к паре $\left(S_{>}, K_{>}\right)$.

Ясно, что на шаге 2 при достаточно малом объеме выхода рекурсию можно остановить. Мы отмечаем эту возможность только ради упрощения анализа алгоритма, а также чтобы сделать алгоритм более общим (так что при $r=n$ алгоритм превращается в QUICKSORT).

\section{4. Анализ алгоритма}

Рассмотрим время работы предложенного алгоритма. Сначала докажем, что с большой вероятностью глубина рекурсии есть $O(\log n)$. Затем покажем, что с большой вероятностью его время работы есть $O(n \log r)$.

Фиксируем некоторый элемент $t \in S$ являющийся целью, и пусть интервалы, содержащие $t$ при реализации алгоритма, суть $I_{0}^{t}, I_{1}^{t}, I_{2}^{t}, \ldots$ размеров $n_{0}^{t}=n, n_{1}^{t}, n_{2}^{t}, \ldots$ Далее мы отбрасываем верхний индекс $t$. На $j$-м шаге разбиения выбранный случайно разделяющий элемент $s_{j}$ разбивает интервал $I_{j}$ на два интервала, одним из которых является $I_{j+1}$. Вероятность того, что $n_{j+1} \geqslant 3 n_{j} / 4$, равна вероятности того, что $s_{j}$ окажется среди $\left\lfloor n_{j} / 4\right\rfloor$ наименьших или наибольших элементов $I_{j}$. Не ограничивая общности, предположим, что $n \equiv 1(\bmod 4)$. В таком случае $n_{j+1} \leqslant 3 n_{j} / 4$ тогда и только тогда, когда $s_{j}$ лежит не далее чем $(n-1) / 4$ от медианы. Отсюда следует, что вероятность того, что $n_{j+1} \leqslant 3 n_{j} / 4$ есть

$$
\frac{1+2(n-1) / 4}{n}=\frac{n+1}{2 n}>\frac{1}{2} .
$$

Пусть $d=16 \ln (4 / 3)+4$. Далее $\lg x$ означает $\log _{4 / 3} x$.

Лемма 1. Для последовательности интервалов $I_{0}, I_{1}, I_{2}, \ldots$ после $d m$ шагов разбиения $\left|I_{d m}\right| \leqslant(3 / 4)^{m} n$ с вероятностью, не меньшей $1-c_{1}(4 / 3)^{-2 m}$, где $c_{1}-$ положительная постоянная, алгоритм останавливается после $d \lg n$ шагов разбиения с вероятностью $1-c_{2} / n$, где $c_{2}-$ положительная постоянная.

Доказательство. Шаг разбиения считается успешным, если он уменьшает размер каждого из индуцированных интервалов не меньше, чем до 3/4 исходного размера. Так как с каждым успешным шагом размер интервала уменьшается до $3 / 4$ исходного размера, число успешных событий не превосходит $m$. Поэтому при не слишком малом $d$ достаточно показать, что число неудач превосходит $d m-m$ с пренебрежимо малой вероятностью. 
Для $j, 0 \leqslant j<d m$, введем индикаторы $X_{j}$, полагая $X_{j}=1$, если $n_{j+1} \geqslant 3 n_{j} / 4$, и $X_{j}=0$, если $n_{j+1}<3 n_{j} / 4$. Положим

$$
X=\sum_{j}^{d m-1} X_{j}
$$

Ясно, что $X_{j}, j=0,1, \ldots, d m-1$, независимы. Как показано выше, $\mathbf{P}\{X j=1\} \leqslant 1 / 2$, $j=0,1, \ldots, d m-1$, откуда следует, что

$$
\mu=\mathbf{E} X=\sum_{J}^{d m-1} \mathbf{E} X_{j}=\sum_{J}^{d m-1} \mathbf{P}\left\{X_{j}=1\right\} \leqslant \frac{d m}{2} .
$$

Используя неравенство Чернова, получаем оценку

$$
\mathbf{P}\{X \geqslant(1+\delta) \mu\} \leqslant \exp \left(-\mu \delta^{2} / 4\right), \quad 0<\delta<2 e-1
$$

которую применим для получения верхней оценки для числа неудач. Мы оценим вероятность $\mathbf{P}\{X \geqslant d m-m\}$. Именно,

$$
\begin{aligned}
\mathbf{P}\{X \geqslant d m-m\} & =\mathbf{P}\{X \geqslant(2-2 / d)(d m / 2)\}=\mathbf{P}\{X \geqslant(1+(1-2 / d))(d m / 2)\} \\
& \leqslant \exp \left(\frac{-(d m / 2)(1-2 / d)^{2}}{4}\right)=\exp \left(\frac{-m(d-4+4 / d)}{8}\right) \\
& =\exp \left(\frac{-m(d-4)}{8}\right)=\exp \left(\frac{-m(16 \ln (4 / 3))}{8}\right) \\
& =e^{2 m \ln (4 / 3)}=(4 / 3)^{-2 m} .
\end{aligned}
$$

Следовательно,

$$
\mathbf{P}\left\{\left|I_{d m}\right| \leqslant(3 / 4)^{m} n\right\} \geqslant \mathbf{P}\{X<d m-m\} \geqslant 1-(4 / 3)^{-2 m} \text {. }
$$

Так как алгоритм останавливается, когда размер активных интервалов становится равным единице, полагая $m=\lg n$, получаем, что

$$
\begin{aligned}
\mathbf{P}\left\{\left|I_{d \lg n}\right| \leqslant 1\right\} & =\mathbf{P}\left\{\left|I_{d \lg n}\right| \leqslant(3 / 4)^{\lg n} n\right\} \\
& \geqslant \mathbf{P}\{X<d \lg n-\lg n\} \\
& \geqslant 1-(4 / 3)^{-2 \lg n}=1-n^{-2} .
\end{aligned}
$$

Так как число целей (а значит и интервалов) может быть равным $\Omega(n)$, используя неравенство Буля, находим, что алгоритм остановится после $d \lg n$ шагов разбиения с вероятностью, не меньшей $1-c / n$, где $c-$ положительная постоянная. 
Теорема 1. Время работы алгоритма есть $O(n \log r)$ с вероятностью, не меньщей $1-c / n$, где $c$ - положительная постоянная.

Доказательство. Алгоритм имеет две фазы. Первая фаза состоит из первых $\lceil\log r\rceil$ итераций, а остальные итерации составляют вторую фазу. Первая фаза состоит в основном из первых $\lceil\log r\rceil$ итераций алгоритма QUICKSORT, в то время как вторая фаза в основном выполняет алгоритм QUICKSELECT. В конце первой фазы число интервалов равно $r+1$, среди которых не более $r$ активных. В течение второй фазы число активных интервалов также не превосходит $r$. При каждой итерации, включая и итерации первой фазы, активный интервал $I$ разбивается на два интервала. Если оба интервала оказываются активными, они сохраняются, в противном случае один из интервалов отбрасывается. Так, при $q>0$ после $2^{q} \log r$ итераций $O\left(r^{q}\right)$ интервалов будет отброшено и не более $r$ интервалов останется.

Ясно, что среднее время работы алгоритма на первой фазе есть $O(n \log r)$ и можно показать, что это происходит с вероятностью очень близкой к единице. Для оценки времени работы на второй фазе используем лемму 1. В этом случае, с высокой вероятностью, проводится не более $d \lg n-\log r$ итераций с не более чем $r$ интервалами, общее число элементов в которых в начале второй фазы не более $n$. Согласно лемме 1 с высокой вероятностью число сравнений на второй фазе оценивается сверху величиной

$$
\begin{aligned}
\sum_{t=1}^{r} \sum_{j}^{d \lg n-\log r}\left(\frac{3}{4}\right)^{j}\left|I_{\log r}^{t}\right| & =\sum_{t=1}^{r}\left|I_{\log r}^{t}\right| \sum_{j}^{d \lg n-\log r}\left(\frac{3}{4}\right)^{j} \\
& <n \sum_{i=0}^{\infty}\left(\frac{3}{4}\right)^{j}=4 n
\end{aligned}
$$

Следовательно, время, затрачиваемое алгоритмом на второй фазе есть $O(n)$. В результате общее время работы алгоритма есть $O(n \log r)$ с вероятностью, большей чем $1-c / n$, где $c$ - положительная постоянная. Как и для многих рандомизированных алгоритмов, эта вероятностная граница может быть улучшена путем уточнения константы $c$.

\section{5. Заключение}

В этой работе для задачи множественного выбора рассмотрен эффективный рандомизированный алгоритм QUICK-MULTISELECTION, время работы которого есть $O(n \log r)$ с вероятностью, не меньшей чем $1-c / n$, где $c$-положительная постоянная. Алгоритм QUICK-MULTISELECTION можно рассматривать как алгоритм осуществляющий единый подход к сортировке, выбору и множественному выбору, так как он вырождается в алгоритм QUICKSELECT при $r=1$ и в алгоритм QUICKSORT при $r=n$. Ясно, что на практике алгоритм, как и другие алгоритмы множественного выбора, следует использовать для решения последней задачи при условии, что $r$ не слишком велико, то есть, если $r$ есть $O\left(n^{\varepsilon}\right)$ при достаточно малом $\varepsilon$.

Автор благодарен Университету имени Короля Фахда за постоянную поддержку. Автор благодарит рецензента за полезные замечания. 


\section{Список литературы}

1. Akl S. G., The design and analysis of parallet algorithms. Prentice Hall, Englewood Cliffs, 1989.

2. Alsuwaiyel M. H., An optimal parallel algorithm for the multiselection problem. Parallel Computing (2001) 27(6), 861-865.

3. Alsuwaiyel M. H., An efficient and adaptive algorithm for multiselection on the PRAM. Proc. ACIS 2nd Intern. Conf. Software Engineering, Artificial Intelligence, Networking and Parallel Computing. Japan, 2001, 140-143.

4. Blum M., Floyd R. W., Pratt V. R., Rivest R. L., Tarjan R. E., Time bounds for selection. J. Computer System Sci. (1973) 7, 448-461.

5. Floyd R. W., Rivest R. L., Expected time bounds for selection. Commun. ACM (1975) 18, 165-172.

6. Fredman M. L., Spencer T. H., Refined complexity analysis for heap operations. J. Computer System Sci. (1987) 21, 269-284.

7. Hoare C. A. R., FIND (Algorithm 65). Commun. ACM (1961) 4, 321-322.

8. Motwani R., Rajhavan P., Randomized Algorithms. Cambridge Univ. Press, Cambridge, 1995.

9. Shen H., Optimal parallel multiselection on EREW PRAM. Parallel Computing (1997) 23, 19871992.

10. Shen H., Efficient parallel multiselection on hypercubes. Proc. 1997 Intern. Symp. on Parallel Architectures, Algorithms and Networks. IEEE CS Press, 1997, pp. 338-342.

11. Shen H., Optimal multiselection in hypercubes. Parallel Algorithms Appl. (2000) 14, 203-212.

12. Shen H., Han Y., Pan Y., Evans D. J., Optimal parallel algorithms for multiselection on meshconnected computers. Intern. J. Comput. Math. (2003) 80, 165-179.

13. Shen H., Chin F., Selection and multiselection on multidimensional meshes. Proc. Intern. Conf. on Parallel and Distributed Processing Techn. and Appl. 2002, 899-906.

Статья поступила 13.05.2004. 